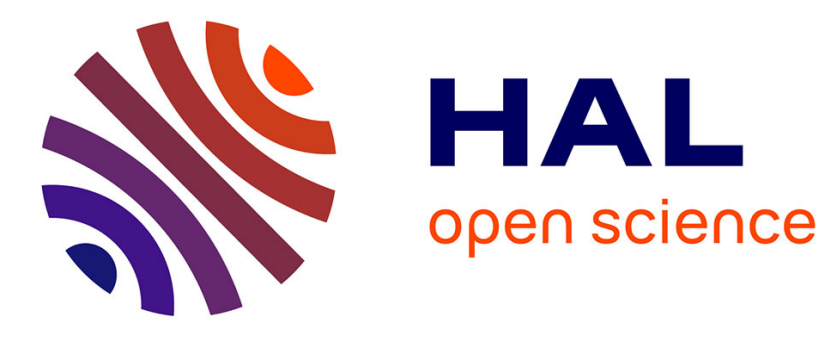

\title{
Status of Virgo
}

F. Acernese, M. Alshourbagy, P. Amico, F. Antonucci, S. Aoudia, P. Astone, S. Avino, L. Baggio, G. Ballardin, F. Barone, et al.

\section{To cite this version:}

F. Acernese, M. Alshourbagy, P. Amico, F. Antonucci, S. Aoudia, et al.. Status of Virgo. 7th Edoardo Almadi Conference on Gravitational Waves (Amaldi7) 18th International Conference on General Relativity and Gravitation (GRG18), Jul 2007, Sydney, Australia. pp.114045, 10.1088/02649381/25/11/114045 . in2p3-00280667

\section{HAL Id: in2p3-00280667 https://hal.in2p3.fr/in2p3-00280667}

Submitted on 19 May 2008

HAL is a multi-disciplinary open access archive for the deposit and dissemination of scientific research documents, whether they are published or not. The documents may come from teaching and research institutions in France or abroad, or from public or private research centers.
L'archive ouverte pluridisciplinaire HAL, est destinée au dépôt et à la diffusion de documents scientifiques de niveau recherche, publiés ou non, émanant des établissements d'enseignement et de recherche français ou étrangers, des laboratoires publics ou privés. 


\section{Status of Virgo}

F.Acernese ${ }^{7,9}$, M.Alshourbagy ${ }^{15,16}$, P.Amico ${ }^{13,14}$, F.Antonucci $^{19}{ }^{19}$ S.Aoudia $^{10}$, P.Astone ${ }^{19}$, S.Avino ${ }^{7,8}$, L.Baggio $^{1}$, G.Ballardin ${ }^{2}$, F.Barone ${ }^{7,9}$, L.Barsotti ${ }^{15,16}$, M.Barsuglia ${ }^{11}$, Th.S.Bauer ${ }^{21}$, S.Bigotta ${ }^{15,16}$, S.Birindelli $^{15,16}$, M.A.Bizouard ${ }^{11}$, C.Boccara ${ }^{12}$, F.Bondu $^{10}$, L.Bosi ${ }^{13}$, S.Braccini ${ }^{15}$, C.Bradaschia ${ }^{15}$, A.Brillet ${ }^{10}$, V.Brisson ${ }^{11}$, D.Buskulic ${ }^{1}$, G.Cagnoli ${ }^{3}$, E.Calloni ${ }^{7,8}$, E.Campagna $^{3,5}{ }^{\text {, F.Carbognani }}{ }^{2}$, F.Cavalier ${ }^{11}$, R.Cavalieri ${ }^{2}$, G.Cella $^{15}$, E.Cesarini ${ }^{3,4}$, E.Chassande-Mottin ${ }^{10}$, A.-C.Clapson ${ }^{11}$, F.Cleva ${ }^{10}$, E.Coccia ${ }^{23,24}$, C.Corda ${ }^{15,16}$, A.Corsi $^{19}$, F.Cottone ${ }^{13,14}$, J.-P.Coulon ${ }^{10}$, E.Cuoco ${ }^{2}$, S.D'Antonio $^{23}$, A.Dari ${ }^{13,14}$, V.Dattilo ${ }^{2}$, M.Davier ${ }^{11}$, R.De Rosa $^{7,8}$, M.Del Prete ${ }^{15,17}$, L.Di Fiore ${ }^{7}$, A.Di Lieto ${ }^{15,16}$, M.Di Paolo Emilio ${ }^{23,25}$, A.Di Virgilioo ${ }^{15}$, M.Evans ${ }^{2}$, V.Fafone ${ }^{23,24}$, I.Ferrante ${ }^{15,16}$, F.Fidecaro ${ }^{15,16}$, I.Fiori ${ }^{2}$, R.Flaminio $^{6}$, J.-D.Fournier ${ }^{10}$, S.Frasca ${ }^{19,20}$, F.Frasconi ${ }^{15}$, L.Gammaitoni ${ }^{13,14}$, F.Garufi ${ }^{7,8}$, E.Genin ${ }^{2}$, A.Gennai ${ }^{15}$, A.Giazotto $^{2,15}$, L.Giordano ${ }^{7,8}$, V.Granata ${ }^{1}$, C.Greverie ${ }^{10}$, D.Grosjean ${ }^{1}$, G.Guidi ${ }^{3,5}$, S.Hamdani ${ }^{2}$, S.Hebri ${ }^{2}$, H.Heitmann ${ }^{10}$, P.Hello ${ }^{11}$, D.Huet ${ }^{2}$, S.Kreckelbergh ${ }^{11}$, P.La Penna $^{2}$, M.Laval ${ }^{10}$, N.Leroy ${ }^{11}$, N.Letendre ${ }^{1}$, B.Lopez ${ }^{2}$, M.Lorenzini $^{3,4}$, V.Loriette ${ }^{12}$, G.Losurdo ${ }^{3}$, J.-M.Mackowski ${ }^{6}$, E.Majorana ${ }^{19}$, C.N.Man ${ }^{10}$, M.Mantovani ${ }^{17,16}$, F.Marchesoni ${ }^{13,14}$, F.Marion ${ }^{1}$, J.Marque $^{2}$, F.Martelli ${ }^{3,5}$, A.Masserot ${ }^{1}$, F.Menzinger ${ }^{2}$, L.Milano $^{7,8}$, Y.Minenkov ${ }^{23}$, C.Moins ${ }^{2}$, J.Moreau ${ }^{12}$, N.Morgado $^{6}$, S.Mosca ${ }^{7,8}$, B.Mours ${ }^{1}$, I.Neri ${ }^{13,14}$, F.Nocera ${ }^{2}$, G.Pagliaroli $^{23}$, C.Palomba ${ }^{19}$, F.Paoletti ${ }^{2,15}$, S.Pardi ${ }^{7,8}$, A.Pasqualetti ${ }^{2}$, R.Passaquieti ${ }^{15,16}$, D.Passuello ${ }^{15}$, F.Piergiovanni $^{3,5}$, L.Pinard ${ }^{6}$, R.Poggiani ${ }^{15,16}$, M.Punturo $^{13}$, P.Puppo ${ }^{19}$, P.Rapagnani ${ }^{19,20}$, T.Regimbau ${ }^{10}$, A.Remillieux ${ }^{6}$, F.Ricci ${ }^{19,20}$, I.Ricciardi ${ }^{7,8}$, A.Rocchi ${ }^{23}$, L.Rolland $^{1}$, R.Romano ${ }^{7,9}$, P.Ruggi ${ }^{2}$, G.Russo ${ }^{7,8}$, S.Solimeno $^{7,8}$, A.Spallicci ${ }^{10}$, M.Tarallo ${ }^{15,16}$, R.Terenzi ${ }^{23}$, A.Toncelli ${ }^{15,16}$, M.Tonelli ${ }^{15,16}$, E.Tournefier ${ }^{1}$, F.Travasso ${ }^{13,14}$, C.Tremola ${ }^{15,16}$, G.Vajente ${ }^{18,16}$, J.F.J. van den Brand $^{21,22}$, S. van der Putten ${ }^{21}$, D.Verkindt ${ }^{1}$, 


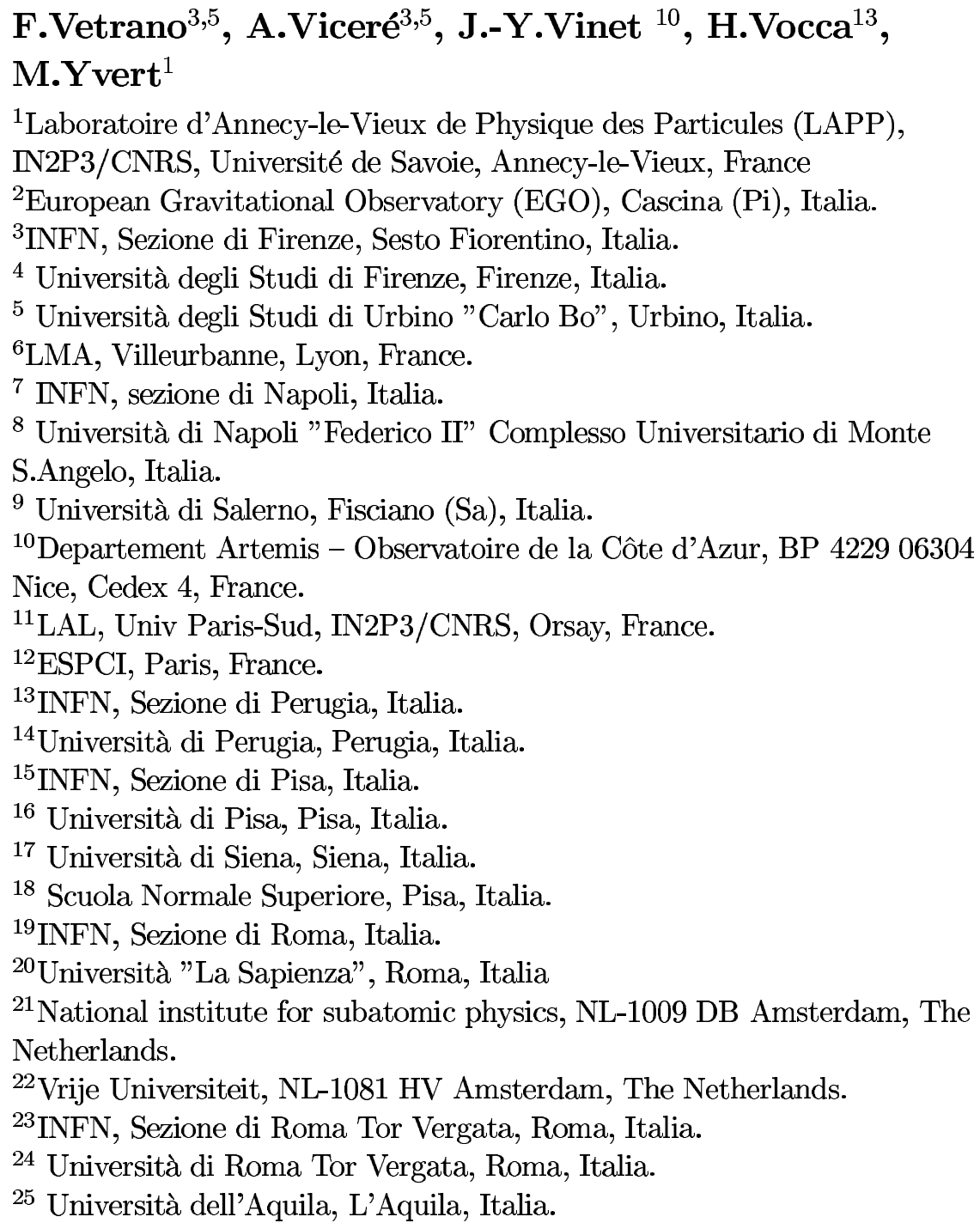

\begin{abstract}
The Virgo detector has now finished its first science run; a science mode duty cycle of more than $80 \%$ and a $4.5 \mathrm{Mpc}$ horizon distance for binary neutron star inspiral sources were achieved. Commissioning breaks were organized during the run which permitted to improve the sensitivity and the robustness of the interferometer against environmental perturbations like bad weather and earthquakes. The post-run commissioning phase has now started, with the goal of preparing the next upgrade step of the detector, Virgo+.
\end{abstract}

\title{
Introduction
}

Virgo [1] is one of the large scale, Earth based, interferometric gravitational wave detectors now under operation $[2,3,4]$. The Virgo collaboration has made significant progress in the commissioning of the instrument over the recent years, that allowed a first science run of the experiment (VSR1) [5] lasting more than four months in 2007 at a sensitivity close to the design sensitivity. The data collected during this run are being analyzed jointly with the LIGO Scientific 
Collaboration, as a full data sharing agreement has been concluded between the two collaborations. After VSR1, Virgo resumed commissioning work to further improve the detector sensitivity. Plans for the future include a shutdown in 2008, to upgrade the detector to the Virgo+ configuration [6], aiming at a sensitivity exceeding the Virgo design one for a subsequent second science run starting in 2009. Another major upgrade is being planned for 2011, to bring the instrument at the level of a second generation detector, the so-called Advanced Virgo [7] configuration.

The paper is organized as follows. The first section is devoted to a summary of the VSR1 run. Section 2 focuses on the achievements of past and current commissioning activities. In section 3 highlights of the data analysis are presented. Finally in section 4 the plans for future upgrades are discussed.

\section{The first Virgo scientific run}

The VSR1 run took place from May 18th to October 1st, 2007. The sensitivity of the Virgo detector at the end of the run is shown in figure 1. Since VSR1 was taken in coincidence with the last months of the fifth science run (S5) of the LIGO and GEO detectors, and Virgo and the LSC have agreed to share data and analyze them jointly, the sensitivity is shown together with that of the LIGO and GEO detectors at the time of S5.

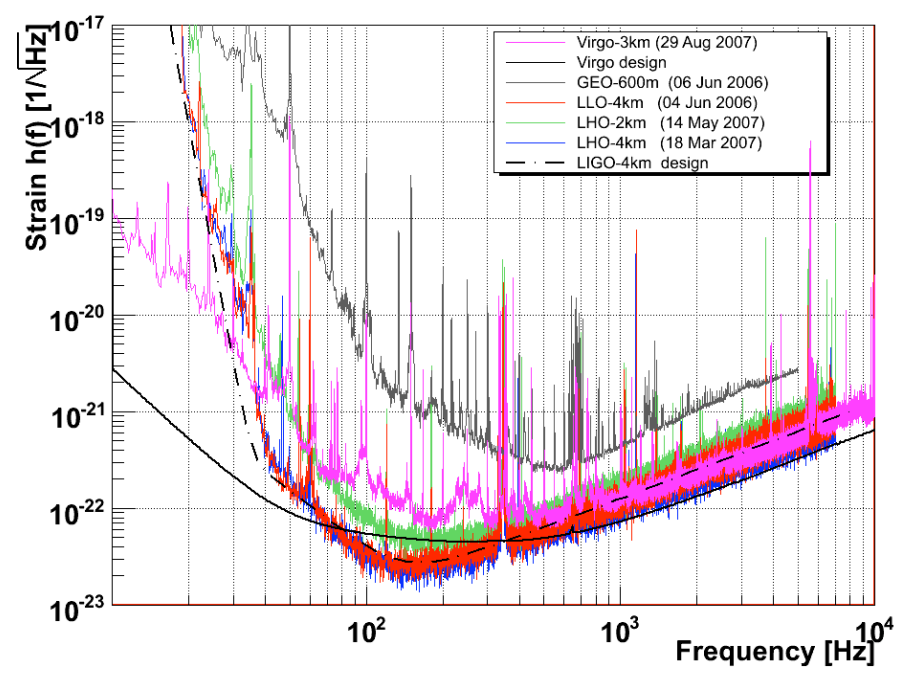

Figure 1. Sensitivity of the Virgo detector at the end of the VSR1 run, shown together with the sensitivities of the LSC detectors at the time of the S5 run.

It is common to express the detector sensitivity as a single number representing the distance of a coalescing binary neutron star system whose signal would be seen with a signal to noise ratio of 8 , averaged over the source sky position, inclination and polarization. Figure 2 shows how this average horizon 
distance evolved during VSR1. Typical values were $3.5 \mathrm{Mpc}$ at the beginning of the run, and more than $4 \mathrm{Mpc}$ at the end of the run. More details will be given in section 2 about the improvements of the instrument and its understanding to achieve this sensitivity enhancement.

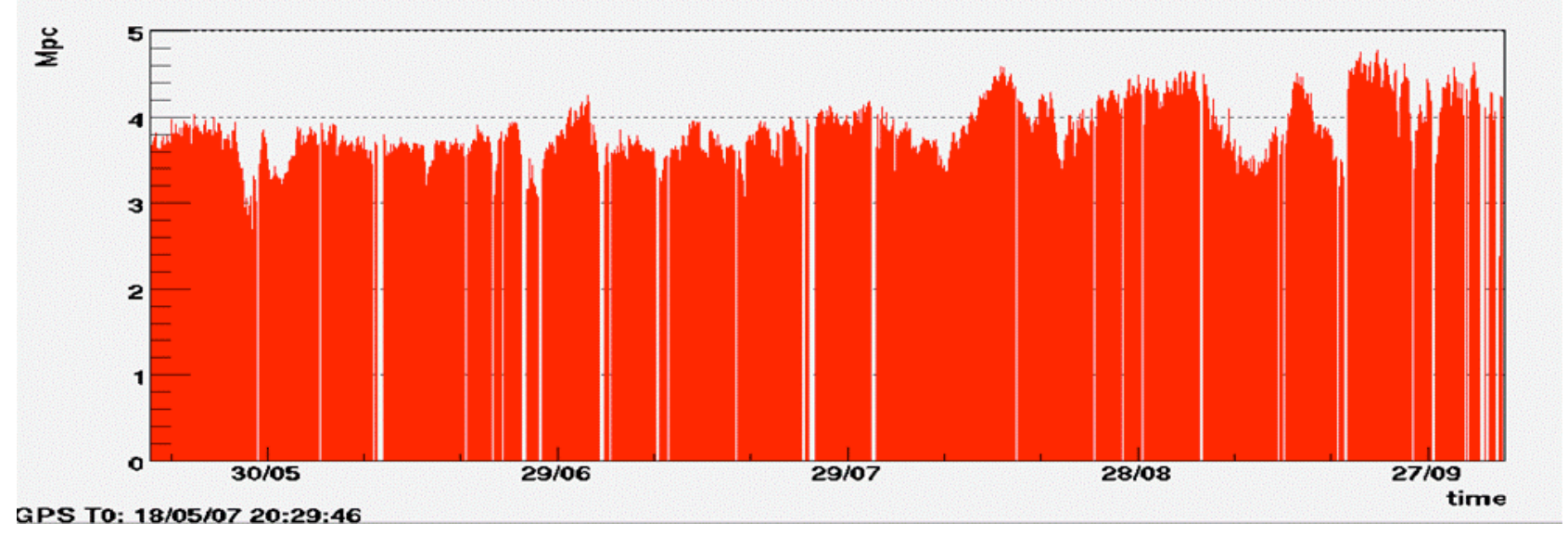

Figure 2. Evolution during the VSR1 run of the horizon distance for coalescing binary neutron star sources (averaged over the source sky position, inclination and polarization).

The noise budget of the detector after the end of the VSR1 run is shown in figure 3. The sensitivity was limited by shot noise above $500 \mathrm{~Hz}$, by longitudinal control noise below $50 \mathrm{~Hz}$, and by excess noise in the intermediate frequency band, believed to be mostly environmental noise.

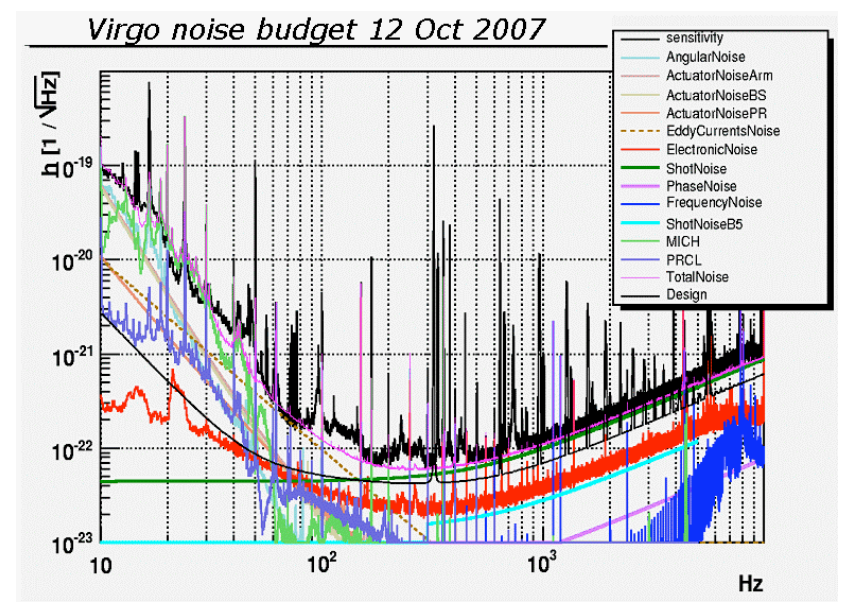

Figure 3. Noise budget of the Virgo detector after the VSR1 run.

The duty cycle of the detector during VSR1 was quite good: the interferometer was locked for $84.2 \%$ of the time, and in science mode for $81.0 \%$ of the time. This high efficiency was achieved because it was usually possible to keep the detector locked for a long time: there were 20 locks longer than 40 
hours, with the longest lock 94 hours long. The main reasons why locks were broken include earthquakes and technical problems at the level of the injection system (loop instabilities of the frequency pre-stabilization system) and the global control system (software crashes). Successful actions were taken during the run to reduce the frequency or impact of those disturbances.

\section{Past and current commissioning activities}

Thermal transient. After the $\mathrm{C} 7$ commissioning run (Sept. 2005) the installation of a Faraday isolator on the suspended injection bench [8] allowed a ten-fold increase of the laser power without disturbing the control systems by back-reflected light. This power increase reduced the shot noise limit and improved the high frequency sensitivity, but it also brought new problems due to heating of the Fabry-Perot cavity input mirrors: cavity parameters change over a time scale of 15-20 minutes after lock acquisition, and science mode cannot be reached before this thermal transient is finished (see figure 4), so that after every unlock almost half an hour of science mode or commissioning time is lost. In order keep the control systems working during the thermal transient, the locking procedure had to adapt the parameters of the control systems to the changing interferometer conditions: alignment loops for different degrees of freedom are switched on at determinate moments, and the locking loops have slow servos measuring and compensating permanently the thermally caused changes in gain for avoiding unlocks due to loop oscillations. The resulting lock acquisition procedure is reasonably stable, with occasional not understood unlocks, but for reducing the time required for reaching science mode, a thermal compensation system will be installed.

Robustness issues. The robustness of the Virgo lock is very dependent on environmental factors: difficult weather conditions (sea waves, wind) reduce the sensitivity by introducing mirror oscillations in the region below $1 \mathrm{~Hz}$, and earthquakes of magnitude above 6 or 6.5 anywhere on the world can cause an unlock. Therefore considerable effort was invested in the improvement of the suspension control system by linking the control of the interferometer mirrors to the input mirrors, instead of having independent local sensors on each mirror. The resulting differential control (Global Inverted Pendulum Control) gave a 2-3 fold increase in robustness against earthquake caused unlocks during VSR1.

Noise reduction. The search for control noise and its elimination was facilitated by the implementation of automated noise budget measurements; after determining the transfer function from a control signal to the gravitational wave signal by injecting colored noise, a measurement of the control noise spectrum can be used for determining its contribution to the dark fringe. Figure 5 shows a recent noise budget for the longitudinal control systems: The short Michelson control system limits the sensitivity at low frequencies by introduction of environmental noise. Since this noise is visible on the photodiode which gives 


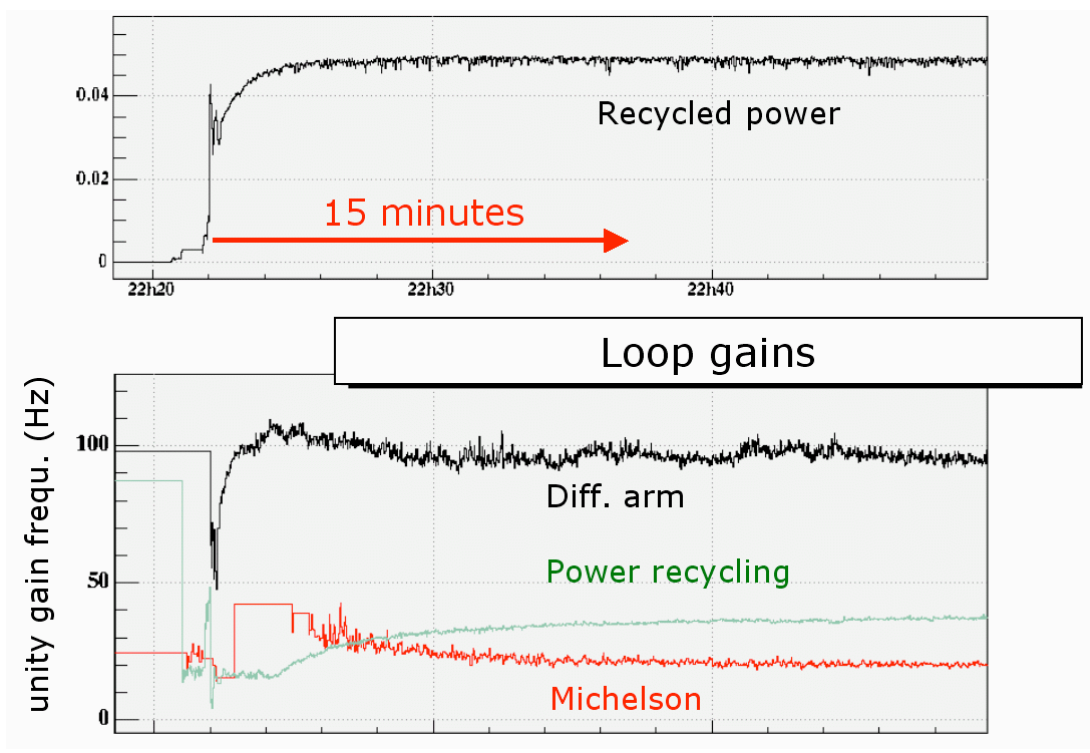

Figure 4. Effects of the thermal transient during lock acquisition. The recycled power undergoes strong changes during the first 5 minutes (upper plot), and the gains of the longitudinal control loops keep changing during 15 minutes (lower plot).

the error signal, one can compensate its effect in the dark fringe by adding it with a negative sign to the control signal for the differential end mirror motion. Using the correct transfer function, the corresponding dark fringe noise can be suppressed by more than 2 orders of magnitude.

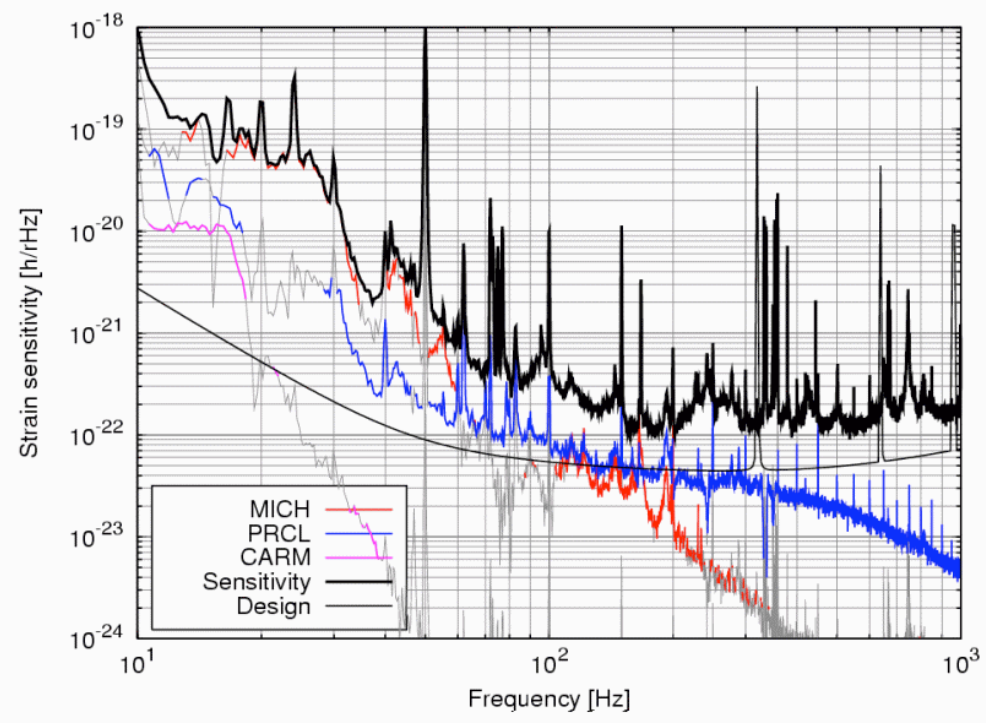

Figure 5. Noise budget for longitudinal control. The noise injected by the power recycling cavity (PRCL), short Michelson (MICH), and common arm length (CARM) controls are shown; these curves include the effect of the noise subtraction technique. 
The noise of the alignment system limits the present sensitivity only below $20 \mathrm{~Hz}$, but it is above the design sensitivity up to $50 \mathrm{~Hz}$. Work on control filters has therefore been done, trying to optimize the trade-off between low frequency gain and high frequency roll-off; this work is presently continuing. Moreover, by better centering of the beam on the mirrors, the coupling of angular noise to the dark fringe is reduced.

Besides control noise, environmental noise is a major limitation for the Virgo sensitivity. These noises are often linked to scattered light; careful elimination of spurious beams and more rigid mounting of optical components helped in their reduction. Before the science run, all optical benches have been isolated from acoustic noise by putting them in acoustic enclosures. Magnetic noise measurements have been performed and several lines in the 50-100 Hz region have been found and eliminated from the spectrum by removing power supplies which had been too close to the mirrors and interacted with the magnets glued on the mirror surface.

Post-science run commissioning. The scope of the commissioning period after the run is the preparation of the Virgo+ upgrade (see section 4) by improving the robustness, stationarity and noise performance of the detector in those areas which will remain unchanged. The first step after the end of the science run was a two-week long detector characterization in run conditions, which allowed verification of the calibration as well as preparing optimization of control loops based on a better knowledge of the detector response. The improvement of the sensitivity, obtained in the last months, makes apparent that the main limitation is now due to environmental noise, so this topic receives a particular attention. Other tasks concern further optimization of longitudinal and angular control systems, reduction of electronics noise in the actuation system, and reduction of thermal effects by cleaning the arm input mirrors in order to reduce absorption of optical power. The residual mirror deformation will be corrected by a thermal compensation system, which will use a $\mathrm{CO}_{2}$ laser for assuring a uniform temperature profile of the input mirrors; this should greatly speed up and simplify the lock acquisition prodecure.

\section{Data analysis}

The Virgo data analysis activities still focus strongly on calibration and detector characterization: generating reliable $h(t)$ data and selecting time periods which can be safely used for search analyses are major tasks. This work benefits from the experience gained with the data taking that took place before VSR1, especially the C7 run (September 2005), and the week-end science runs (WSR1 to WSR10) that occurred in late 2006 and early 2007 [9, 10].

The data taking prior to VSR1 was also the opportunity for the search groups to pursue the development of their analysis methods and pipelines, and to complete some searches $[11,12]$. We mention the example of burst 
searches, where a blind search of the $\mathrm{C} 7$ data [13] and dedicated searches of gravitational wave signals emitted in coincidence with gamma ray bursts have been performed $[14,15]$. Preparing the joint data analysis with the LSC has received also a lot of attention [16].

\section{Future upgrades of the detector}

We expect to start a second scientific run in 2009, with an upgraded version of the detector, namely the Virgo+ configuration. Virgo+ is meant to be a step on the upgrade path that will bring Virgo to the level of a second generation detector, in the Advanced Virgo configuration. The upgrades for Virgo+ are going to start in the first half of 2008: increase of the laser power to $50 \mathrm{~W}$, installation of a thermal compensation system, upgrade of the data acquisition and control electronics.

We are working to install monolithic suspensions in Virgo+, with new mirrors allowing to increase the finesse of the Fabry-Perot cavities to a value of 150 . However we will start with the existing steel wire suspensions and the current mirrors, after cleaning them to reduce absorption. Changing the mirrors while keeping the suspensions could also be a conservative option. The expected sensitivities with each of the possible solutions are shown in figure 6 .

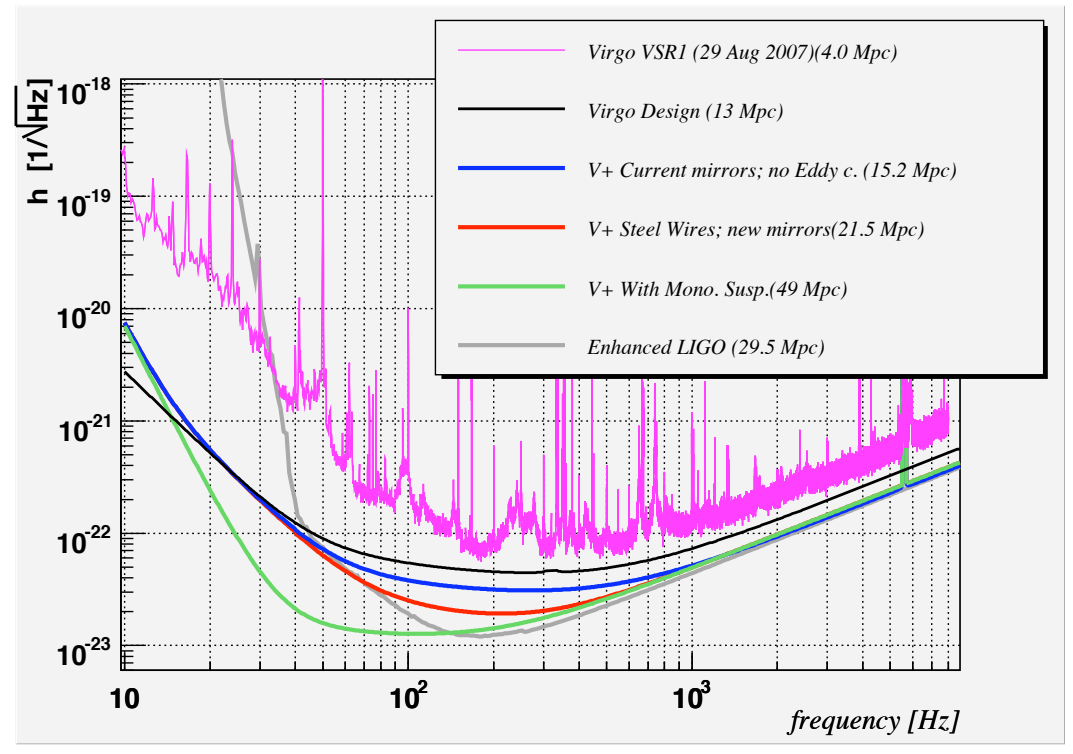

Figure 6. Various options for the sensitivity of Virgo+, corresponding to the different configurations of the mirror suspensions described in the text.

The preparation of Advanced Virgo is now a priority for the collaboration. The goal is to bring a factor 10 improvement in sensitivity with respect to the nominal Virgo. A document describing the Advanced Virgo baseline design [7] has been released, together with preliminary cost and project execution plans, 
and submitted to the funding institutions. If approved, we expect the funding for Advanced Virgo to start in year 2009.

\section{Conclusion}

The first science run of Virgo was a success, with good sensitivity and efficiency. The task of analyzing jointly with the LSC the data taken during VSR1 is now making great strides. In parallel, we are proceeding to upgrade the detector in two steps - Virgo+ and Advanced Virgo.

\section{References}

[1] http://www.virgo.infn.it

[2] http://www.ligo.caltech.edu

[3] http://geo600.aei.mpg.de/

[4] http://tamago.mtk.nao.ac.jp/

[5] VIRGO: a large interferometer for Gravitational Wave detection started its first scientific run Virgo Collaboration, F.Frasconi et al., to be published in Journal of Physics: Conference Series

[6] Virgo upgrades

Virgo Collaboration, M Punturo et al, this issue of Class. Quantum Grav.

[7] Advanced Virgo Conceptual Design

Virgo Collaboration, G.Losurdo et al. 2007 http://wwwcascina.virgo.infn.it/advirgo/docs/AdV_Design.pdf

[8] The VIRGO injection system

Virgo Collaboration, F.Bondu et al, 2002 Class. Quantum Grav. 1918291833

[9] Data quality studies for burst analysis of Virgo data acquired during Weekly Science Runs

Virgo Collaboration, F.Acernese et al, 2007 Class. Quantum Grav. 24 S415-S422

[10] Analysis of noise lines in the Virgo C7 data

Virgo Collaboration, F.Acernese et al, 2007 Class. Quantum Grav. 24 S433-S443

[11] Status of coalescing binaries search activities in Virgo

Virgo Collaboration, F.Acernese et al, to be published in Class. Quantum Grav.

[12] Coincidence analysis between periodic source candidates in C6 and C7 Virgo data

Virgo Collaboration, F.Acernese et al, 2007 Class. Quantum Grav. 24 S491-S499

[13] Gravitational wave burst search in the Virgo C7 data

Virgo Collaboration, in preparation

[14] Gravitational waves by gamma-ray bursts and the Virgo detector: the case of GRB 050915a

Virgo Collaboration, F.Acernese et al, 2007 Class. Quantum Grav. 24 S671-S679

[15] Search for gravitational waves associated with GRB 050915a using the Virgo detector Virgo Collaboration, in preparation

[16] Prospects for stochastic background searches using Virgo and LSC interferometers

G.Cella, C.N.Colacino, E.Cuoco, A.Di Virgilio, T.Regimbau, E.L.Robinson and

J.T.Whelan 2007 Class. Quantum Grav. 24 S639-S648 